\title{
Therapeutic strategy for pineal parenchymal tumor of intermediate differentiation (PPTID): case report of PPTID with malignant transformation to pineocytoma with leptomeningeal dissemination 6 years after surgery
}

\author{
Toshiaki Bando, MD, ${ }^{1}$ Yasushi Ueno, MD, PhD, ${ }^{1}$ Narihide Shinoda, MD, ${ }^{1}$ Yukihiro Imai, MD, PhD, ${ }^{2}$ \\ Kazuhito Ichikawa, MD, PhD, ${ }^{3}$ Yoji Kuramoto, MD, ${ }^{1}$ Takahiro Kuroyama, MD, ${ }^{1}$ Daisuke Shimo, MD, \\ Kazuyuki Mikami, MD, ${ }^{1}$ Shinya Hori, MD, ${ }^{1}$ Masato Matsumoto, MD, PhD, ${ }^{1}$ and \\ Osamu Hirai, MD, PhD'
}

\begin{abstract}
1Department of Neurosurgery and Stroke Center, Shinko Hospital; ${ }^{2}$ Department of Pathology, Kobe City Medical Center General Hospital; and ${ }^{3}$ Department of Pathology, Shinko Hospital, Kobe, Japan

Pineal parenchymal tumor of intermediate differentiation (PPTID) is rare. The WHO first classified PPTID in 2000 as a pineal parenchymal tumor (PPT) with an intermediate prognosis between pineocytoma (PC) and pineoblastoma (PB). It is considered an intermediate-grade tumor and divided into WHO grade II or III.

The number of available reports about PPTID is presently limited, and the appropriate management for this tumor has not yet been determined.

The authors report a rare case of PC in a 63-year-old woman who presented with lower-extremity weakness and gait disturbance. A pineal mass lesion was detected on MRI. A diagnosis of PC was established after microsurgical gross-total tumor resection, and the patient received no adjuvant therapy after surgery. Two years after surgery, a partial recurrence was recognized and Gamma Knife radiosurgery was performed. Fours years later, the patient developed diffuse leptomeningeal dissemination. She was successfully treated with craniospinal irradiation. Leptomeningeal dissemination may develop 6 years after the initial diagnosis of PC. A histopathological study of the recurrent tumor revealed a malignant change from PC to PPTID.
\end{abstract}

The present case shows the importance of long-term follow-up of patients with PPTs following resection and the efficacy of craniospinal irradiation in the treatment of leptomeningeal dissemination.

https://thejns.org/doi/abs/10.3171/2018.2.JNS171876

KEYWORDS pineal parenchymal tumor of intermediate differentiation; leptomeningeal dissemination; oncology

$\mathrm{P}$ INEAL parenchymal tumors (PPTs) are rare, accounting for less than $0.3 \%$ of all CNS tumors. ${ }^{4,13,15,25,27}$ They exhibit remarkable morphological variation, and their histology is subdivided into pineocytoma (PC) (WHO grade I), pineoblastoma (PB) (WHO grade IV), PPT of intermediate differentiation (PPTID) (WHO grade II or III), or papillary tumor of the pineal region (WHO grade II or III), according to the 2007 WHO classification for tumors of the CNS. ${ }^{15,19}$
PPTID is a newly defined CNS tumor, which was first identified by Schild and colleagues ${ }^{26}$ in 1993 and categorized by the WHO in 2000 as PPT with an intermediate prognosis between PC and PB. It is regarded as an intermediate-grade tumor and subdivided into WHO grade II or III.

PPTIDs may account for up to approximately $20 \%$ of PPTs, and PCs and PBs each account for approximately $40 \%$ of PPTs. ${ }^{15,19}$

ABBREVIATIONS NCAM = neural cell adhesion molecule; $\mathrm{PB}=$ pineoblastoma; $\mathrm{PC}=$ pineocytoma; $\mathrm{PPT}=$ pineal parenchymal tumor; $\mathrm{PPTID}=\mathrm{PPT}$ of intermediate differentiation; SRS = stereotactic radiosurgery.

SUBMITTED August 13, 2017. ACCEPTED February 8, 2018.

INCLUDE WHEN CITING Published online July 20, 2018; DOI: 10.3171/2018.2.JNS171876. 

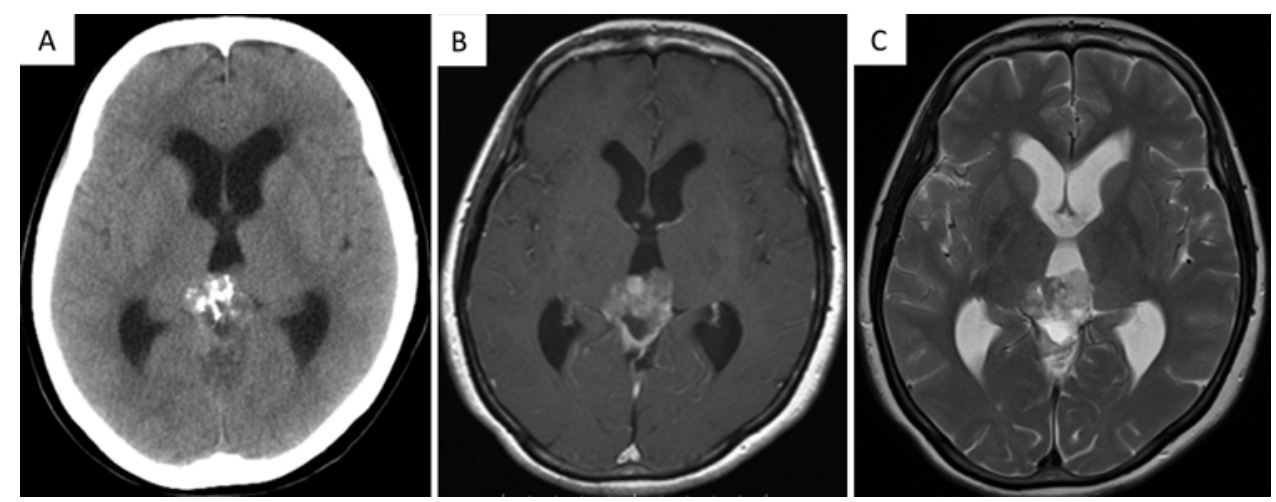

FIG. 1. A: CT scan showing a hyperdense mass in the pineal region with partial calcification. B and C: Gd-enhanced and T2weighted MR images demonstrating a heterogeneously enhancing pineal mass causing hydrocephalus.

Although there is no sex predominance, the incidence of PPTID is somewhat more common among women, teenagers, and middle-aged patients..$^{15,19,24}$ The reported 5 -year survival rates of these tumors are from $86 \%$ to $100 \%$ for PC, $74 \%$ for WHO grade II PPTID, and $39 \%$ for WHO grade III PPTID. ${ }^{24}$

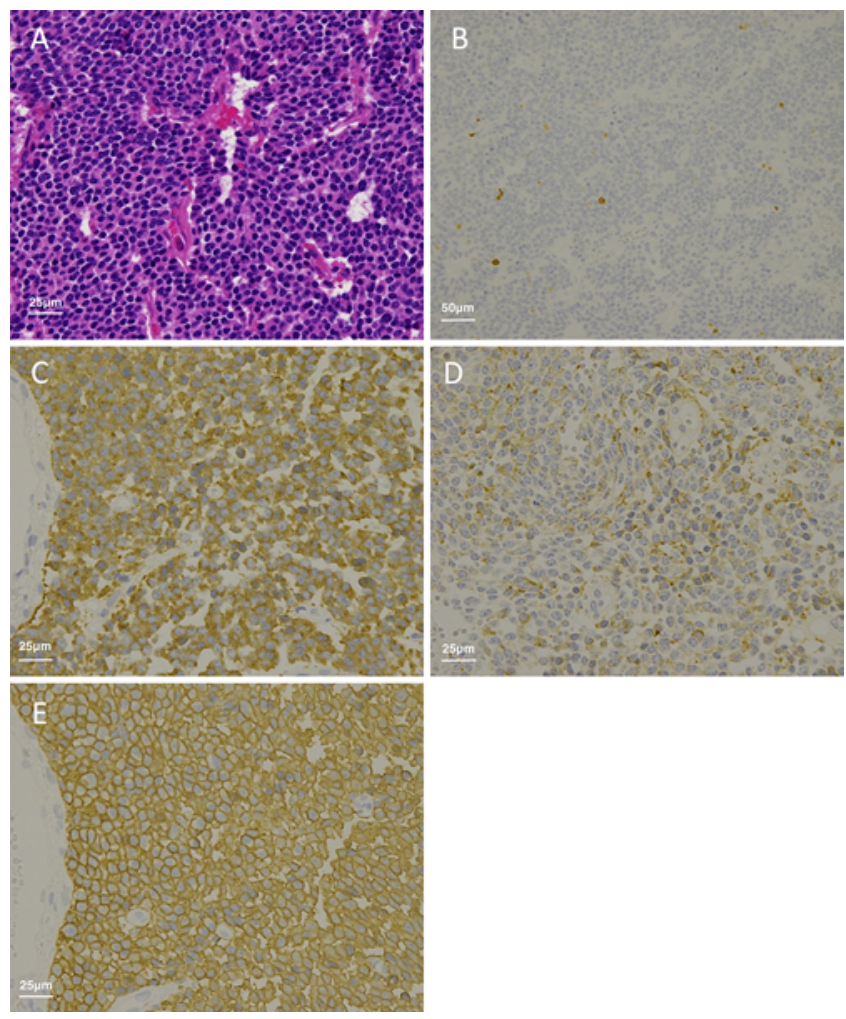

FIG. 2. Histological studies. A: Photomicrograph showing small, uniform, mature cells that virtually lack mitoses, with numerous, large pineocytomatous rosettes. $\mathrm{H} \& \mathrm{E}$, original magnification $\times 400$. B: Study showing an MIB-1 index of $2 \%$. Ki-67, original magnification $\times 200$. C: Synaptophysin-positive result. Original magnification $\times 400$. D: Chromogranin-positive result. Original magnification $\times 400$. E: NCAM-positive result. Original magnification $\times 400$. Figure is available in color online only.
In this study, we report the rare case of a patient with CSF dissemination 6 years after she underwent resection of the PC. Moreover, histopathological examination of the recurrent tumor revealed a malignant change to PPTID.

\section{Case Report}

Presentation

In February 2009, a 63-year-old woman presented with bilateral lower-extremity weakness and gait disturbance. She had no visual field complaints. Her pupils were bilaterally equal and round, and they exhibited a normal reaction to light. Her upward gaze was slightly abnormal, demonstrating evidence of Parinaud's syndrome. There were no significant findings in the patient's own history or family history. Blood tests including any tumor markers were also not significant. CT and MRI revealed a pineal mass with heterogeneous enhancement and partial calcification, causing hydrocephalus (Fig. 1). Leptomeningeal dissemination was not identified on whole-spine MRI. Digital subtraction angiography demonstrated no abnormal vessels or tumor stain.

\section{Operation and Diagnosis}

A craniotomy and microsurgical gross-total tumor resection via a right occipital transtentorial approach were performed. The histopathological examination demonstrated a primary pineal tumor with uniform cells, round-shaped nuclei, and focal clusters of cells consistent with pineocytomatous rosettes. Immunohistochemical examination revealed positive expression of synaptophysin, chromogranin, and neural cell adhesion molecule (NCAM). The MIB-1 index was $2 \%$ (Fig. 2). The final pathological diagnosis was PC. After surgery, no residual tumor was identified on Gd-enhanced MRI. Moreover, all her symptoms and hydrocephalus dramatically improved.

\section{Tumor Recurrence}

Gd-enhanced MRI was performed every 6 months following surgery to identify signs of tumor regrowth and recurrence. In April 2011, recurrence of the pineal lesion was recognized. PC was thought to be less likely to under- 

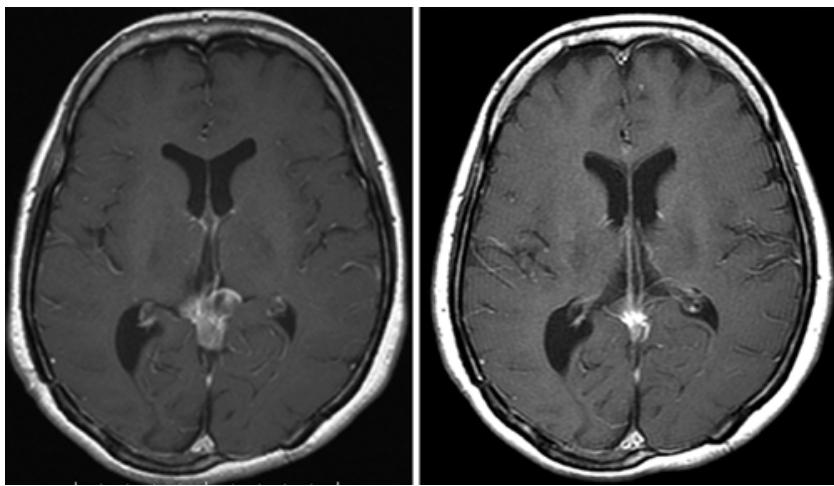

FIG. 3. Left: In April 2011, Gd-enhanced MR image showing partial recurrence of tumor in the pineal region. Right: After SRS in June 2011, Gd-enhanced MR image demonstrating an almost complete resolution of the enhanced tumor region.

go leptomeningeal dissemination compared to PPTID and PB. As such, the patient underwent stereotactic radiosurgery (SRS), which is associated with fewer systemic side effects compared with conventional radiation therapy. The Leksell Gamma Knife Perfexion (Elekta Instruments) was used, and the maximum dose and margin dose were $28 \mathrm{~Gy}$ and 14 Gy, respectively. At the time of SRS, the tumor size was approximately $13 \times 14 \times 20 \mathrm{~mm}$. After treatment, the enhanced region of the tumor almost completely disappeared (June 2011), and complete resolution of the pineal tumor was noted. Regular Gd-enhanced MRI follow-up was scheduled every 4 months (Fig. 3).

\section{Leptomeningeal Dissemination}

In December 2014, almost 6 years after the initial surgery, the patient returned to our hospital complaining of bilateral lower-extremity weakness and dizziness for the previous several months; progressive low-back pain for the past 1 month; and headache, nausea, poor appetite, and fatigue increasing over the previous several weeks.

Whole-brain and whole-spine MRI revealed multiple widespread leptomeningeal enhanced masses involving the cervical and lumbar spine, as well as the lateral ventricle, perimesencephalic cistern, interpeduncular cistern, and basal cistern (Fig. 4).

\section{Diagnosis and Treatment}

Pathological studies of CSF revealed no abnormal cells. Blood and CSF studies revealed no evidence of bacterial infection, such as Cryptococcus, syphilis, tuberculosis, or others. Chest and abdominal CT revealed no evidence of a systemic malignant tumor.

To determine the pathology of the multiple tumors, an endoscopic biopsy of the mass in the left lateral ventricle was performed (Fig. 5). Histopathological studies of the tumor specimen revealed no evidence of a WHO grade IV PB, but the findings were consistent with moderate cellularity, mild to moderate atypical nuclei, and low mitosis $(<6 / 10 \mathrm{hpf})$. Necrosis and endothelial proliferation were absent. Immunohistochemical examination revealed positive expression of synaptophysin and negative expression of chromogranin A, CD3, CD5, CD10, and CD20, and the MIB-1 index was 8\% (Fig. 6). The final pathological diagnosis was PPTID.
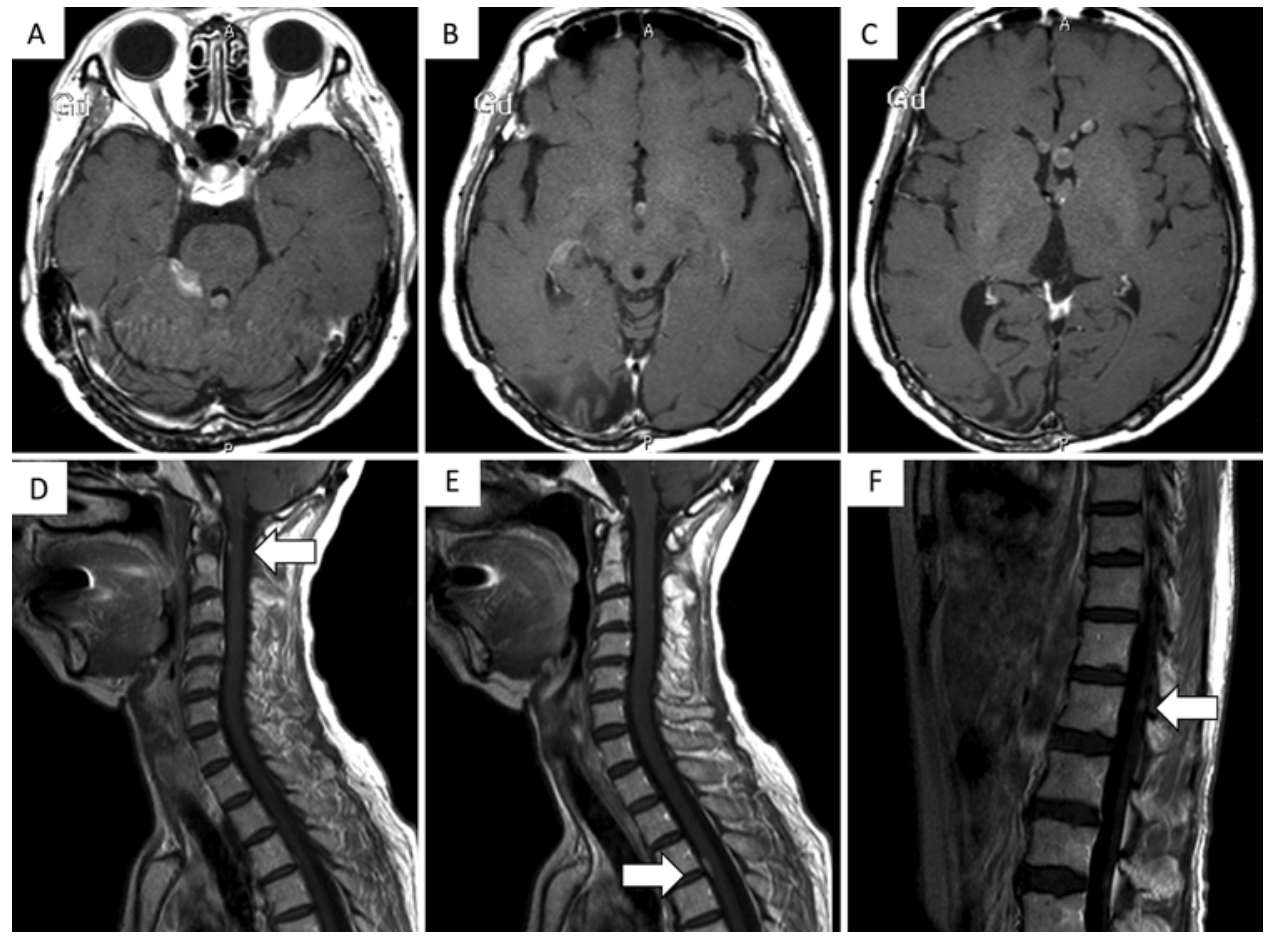

FIG. 4. A-C: Gd-enhanced MR images demonstrating multiple enhanced masses in the lateral ventricle, perimesencephalic cistern, interpeduncular cistern, and basal cistern. D-F: Gd-enhanced MR images demonstrating multiple enhanced masses at the C1 vertebra, the T3-4 vertebra, and the L3-4 vertebra (arrows). 


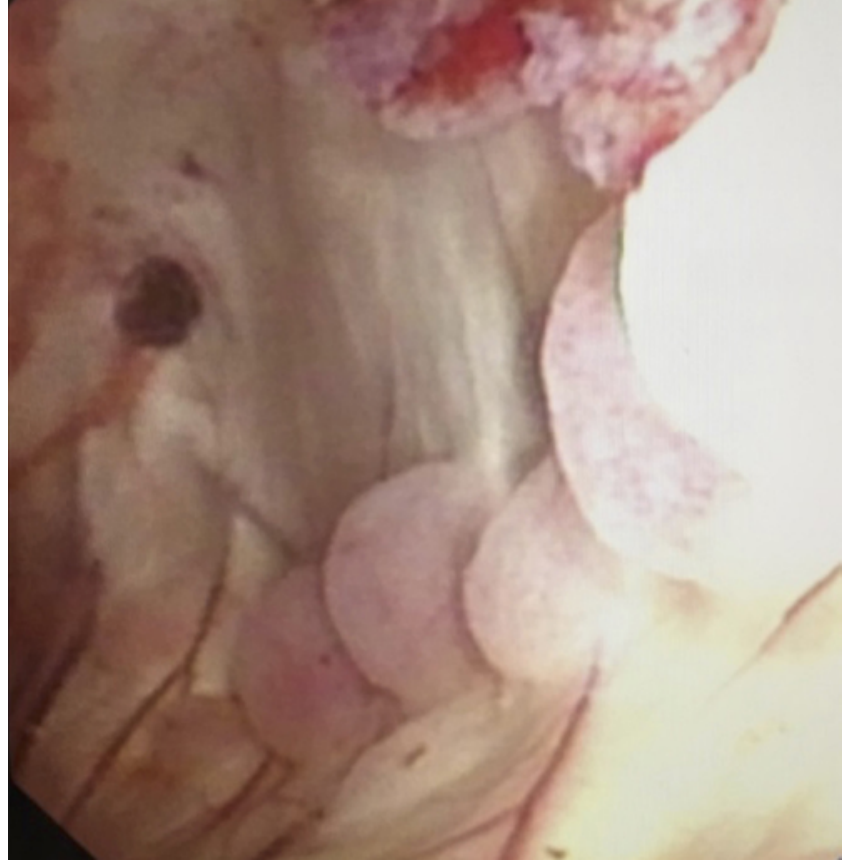

FIG. 5. Endoscopy showed multiple disseminated lesions at the left frontal horn of the lateral ventricle, and a biopsy was performed. Figure is available in color online only.

The patient underwent craniospinal irradiation (total dose 30.6 Gy in 1.8-Gy fractions) to the whole brain and spine. Chemotherapy was also administered (3 courses of ICE therapy [isofamide $900 \mathrm{mg} / \mathrm{m}^{2}$, cisplatin $20 \mathrm{mg} / \mathrm{m}^{2}$, and etoposide $\left.60 \mathrm{mg} / \mathrm{m}^{2}\right]$ ).

\section{Follow-Up}

By March 2015, Gd-enhanced MRI demonstrated the disappearance of all leptomeningeal enhanced masses. The patient's symptoms had almost completely resolved except for her general fatigue.

Two years after craniospinal irradiation with ICE therapy, she remained tumor free. Additionally, no neurological deficits or any late neurological side effects due to radiation necrosis and edema were present.

\section{Discussion}

PPTID is a newly defined CNS tumor. It was first identified by Schild et al. ${ }^{26}$ in 1993 and classified by the WHO in 2000 as a PPT with an intermediate prognosis between $\mathrm{PC}$ and PB. It is regarded as an intermediate-grade tumor and can be subdivided into WHO grade II or III. PPTID may account for up to approximately $20 \%$ PPTs, and PC and PB each account for approximately $40 \%$ of PPTs. ${ }^{15,19}$

Although there is no sex predominance in particular, the incidence is somewhat more common among females, teens, and middle-aged patients. ${ }^{15,19,24}$ The reported 5-year survival rates of these tumors are from $86 \%$ to $100 \%$ for PC, $74 \%$ for WHO grade II PPTID, and $39 \%$ for WHO grade III PPTID. ${ }^{24}$

The optimal treatment for PPTID has not yet been determined since it is a relatively new tumor entity and lim-

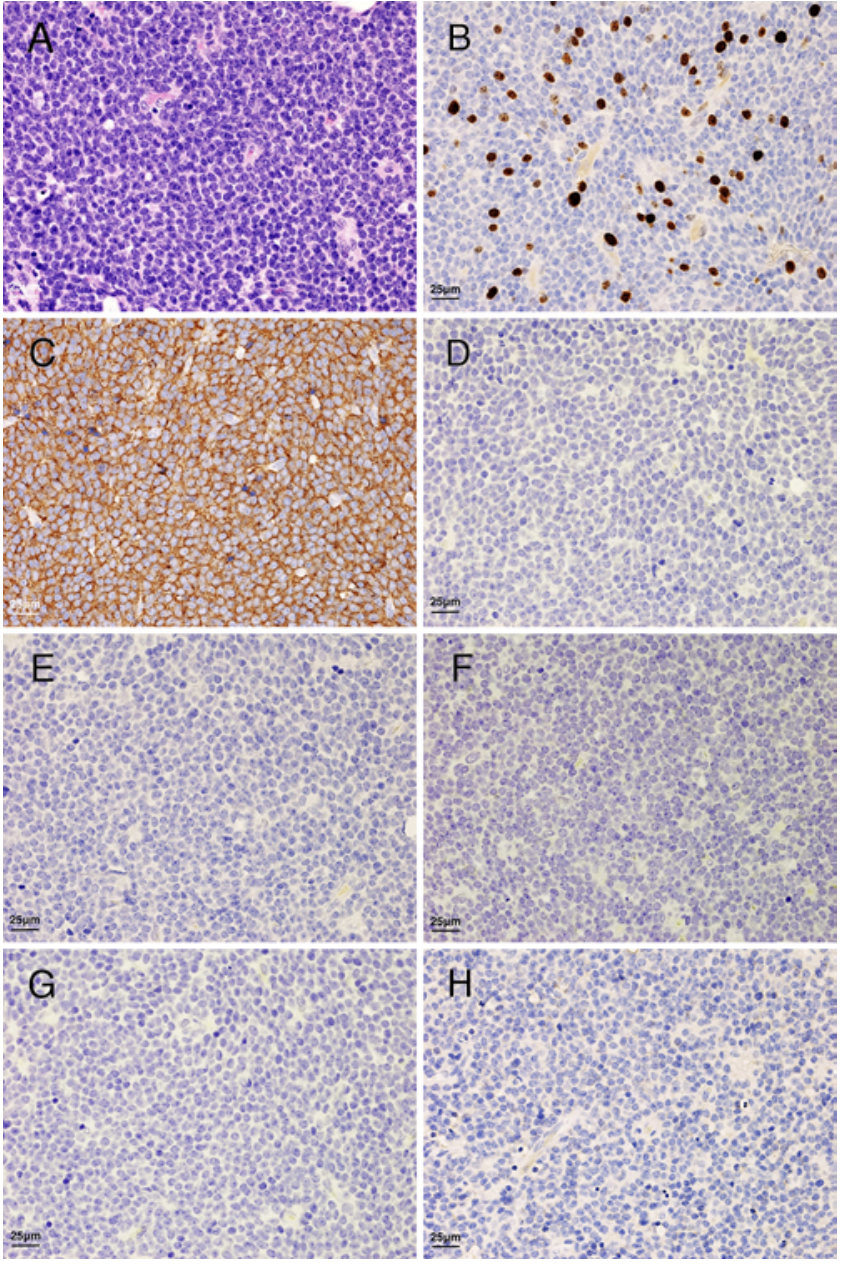

FIG. 6. Histological studies. A: Photomicrograph showing moderate cellularity, mild to moderate atypical nuclei, and low mitoses. $\mathrm{H} \& \mathrm{E}$, original magnification $\times 400$. B: Study showing an MIB-1 index of $8 \%$. Ki- 67 , original magnification $\times 400$. C: Synaptophysin-positive result. Original magnification $\times 400$. D: CD3-negative result. Original magnification $\times 400$. E: CD5-negative result. Original magnification $\times 400$. F: CD10negative result. Original magnification $\times 400$. G: $C D 20$-negative result. Original magnification $\times 400$. $\mathrm{H}$ : Chromogranin A-negative result. Original magnification $\times 400$. Figure is available in color online only.

ited published data are currently available. Furthermore, there have been few publications on PC associated with leptomeningeal dissemination. As such, details about the spread, time course, and optical treatment of diffuse leptomeningeal progression are largely unknown., ${ }^{9,15,26}$

\section{Pathology}

PCs (WHO grade I) are well differentiated with small, uniform, mature cells that virtually lack mitoses with innumerable, large pineocytomatous rosettes. PBs (WHO grade IV) are highly cellular tumors with frequent mitoses, irregular nuclei, and large nucleus-to-cytoplasmic ratios, forming patternless sheets, with necrosis, and rarely forming rosettes. ${ }^{19}$

PPTIDs (WHO grades II and III) are histologically an intermediate between $\mathrm{PC}$ and $\mathrm{PB}$, and generally have 
moderate cellularity, mild to moderate atypical nuclei, and low to moderate mitoses. In contrast to PBs, necrosis and endothelial proliferation are absent.

Sato and Kubota reported that the appearance of necrosis, rate of mitosis, and immunohistochemical expression of neurofilament protein are used to classify PPTIDs as WHO grade II or III. ${ }^{24}$ Jouvet et al. reported that a WHO grade II tumor has $<6$ mitoses and is strongly immunopositive for neurofilaments, while a WHO grade III tumor would have $>6$ mitoses or $<6$ mitoses without strong immunostaining for neurofilaments. ${ }^{15}$

PPTIDs have positive expression of synaptophysin, neurofilament, chromogranin A, and renal S antigen immunohistochemically. The MIB-1 proliferation index ranges from $3 \%$ to $10 \%,{ }^{9}$ which is significantly higher in $\mathrm{PBs} .{ }^{29}$

\section{Treatment of PPTID}

\section{Radiotherapy}

The optimal treatment for PPTID has not yet been determined. PPTID is considered a relatively new tumor and limited reported data are available. It is regarded as a PPT with an intermediate prognosis between PC and PB.

PCs are generally treated with resection. Total or subtotal resection leads to a favorable outcome without adjuvant treatment. ${ }^{5,6}$ Conversely, PBs should be treated with resection and adjuvant treatment containing chemotherapy and craniospinal radiation therapy. More aggressive treatments, such as high-dose myeloablative chemotherapy with stem cell transplant, may be required to treat PBs. ${ }^{8}$

Some reports indicate that PPTID can be treated with surgery alone because PPTID is prognostically similar to PC and shows long-term survival. Other studies have described PPTIDs as tumors with CSF dissemination and recommend postoperative adjuvant therapy similar to that used with PBs, $, 15,26$ since PPTID genomically resembles $\mathrm{PB}$ and is a radiosensitive tumor. ${ }^{1,23}$

Importantly, SRS is an invaluable therapy for patients with PCs. Hasegawa et al. analyzed the cases of 16 patients who had received SRS as the primary or adjuvant treatment for PPT (10 cases of PC, 2 cases of mixed PC$\mathrm{PB}$, and 4 cases of $\mathrm{PB}$ ). The maximum radiation dose varied from 24 to $40 \mathrm{~Gy}$ (mean $30.5 \mathrm{~Gy}$ ), and the margin dose varied from 12 to 20 Gy (mean 15.3 Gy). In 14 patients who underwent follow-up imaging, 4 (29\%) showed complete remission, 8 (57\%) showed partial remission, 2 (14\%) showed no change, and no patient had local dissemination. ${ }^{12}$ The 2-year and 5-year actuarial overall survival rates were $75.0 \%$ and $66.7 \%$, respectively. Compared to conventional radiotherapy, SRS can reduce the radiation dose to surrounding normal brain tissue and augment the radiobiological effect on the specified tumor volume. Therefore, SRS may have a lower risk of additional longterm cognitive morbidity. When local dissemination occurs after SRS, however, additional craniospinal radiation therapy and chemotherapy are required.

In spite of various reports emphasizing the efficacy of irradiation, the extent of irradiation in patients with PPTID has not been determined, and efficacy of craniospinal and whole-ventricular irradiation for patients with PPTID remains a matter of debate. Ito et al. reported 5 cases of PPTID in which the patients exhibited a complete response to radiotherapy. ${ }^{14}$

Based on a clinicopathological study of 76 patients with pineal tumors, Fauchon et al. found that irradiation had no clear influence on patient survival. ${ }^{9}$ Stoiber et al. reported that local irradiation is effective in patients with PC and PPTID. ${ }^{28}$ Das et al. emphasized that irradiation was necessary for patients with PPTID if complete resection was extremely difficult. Also, craniospinal irradiation would be a logically acceptable approach for patients with leptomeningeal dissemination.?

Schild et al. reported on 30 patients with pineal tumors and found a relationship between the radiation dose and survival time in patients with PPT. ${ }^{25}$ They reported that patients receiving a higher dose (>50 Gy) of radiation had a significantly improved 3-year survival rate compared with those receiving a lower dose $(94 \%$ vs $56 \%$, respectively). Finally, Das et al. demonstrated a favorable outcome in patients with PPTID who received a 54-Gy dose of radiation. ${ }^{7}$

\section{Chemotherapy}

Chemotherapy for PPTID also remains a matter of debate. There is currently no standard systemic chemotherapy regimen for PPTID patients. Various combinations of chemotherapy for PPTID patients have been reported: vincristine $\left(0.6 \mathrm{mg} / \mathrm{m}^{2}\right)$, nimustine $\left(60 \mathrm{mg} / \mathrm{m}^{2}\right)$, carboplatin $\left(110 \mathrm{mg} / \mathrm{m}^{2}\right)$, and interferon $\beta\left(3 \times 10^{6} \mathrm{IU}\right),{ }^{30}$ or combination with cisplatin, ifosfamide, etoposide, vinblastine, and methotrexate, such as ICE therapy (isofamide + carboplatin + etoposide) or PCV therapy (procarbazine + lomustine + vincristine). ${ }^{16,17}$

Some molecular-targeted agents showed probable benefit for PPTID patients with a mutation of epidermal growth factor receptor (in-frame deletion of exons 2-7). ${ }^{17}$ PPTID patients may get some benefit from a combination of chemotherapy and molecular-targeted therapy in the near future.

\section{Side Effects of Radiotherapy or Chemotherapy}

Late adverse effects after radiotherapy or chemotherapy are also considerable problems. Craniospinal irradiation combined with systemic chemotherapy increases the risk of long-term side effects, including severe cognitive impairment $4-6$ years after treatment. ${ }^{30}$ Chemotherapy enhances neurocognitive impairment due to injury to neural progenitor cells or neural stem cells. ${ }^{11,18}$ Some molecular-targeted agents including epidermal growth factor receptor tyrosine kinase inhibitors and some proteasome inhibitors are also more neurotoxic. ${ }^{2}$ Moreover, radiation therapy may increase chemotherapeutic neurocognitive toxicity by causing blood-brain barrier damage. ${ }^{3}$ Damage to the blood-brain barrier will likely increase the neurotoxic effect on the brain caused by chemotherapy.

Previous studies have reported an association between radiation dose and injury to neural stem cells and the temporal lobes. Modifying the route of radiation delivery may mitigate the cognitive neurotoxicity of radiation therapy. For example, avoiding radiation dose to the hippocampus during radiation therapy lowers the incidence of long-term cognitive damage in the cases of brain metastases. ${ }^{22}$ 
Watanabe et al. reported on 5 PPTID patients who received both craniospinal and whole-ventricular irradiation. After treatment, damage to the cerebral white matter was revealed in MRI findings that corresponded to neurocognitive impairment. Taking into consideration the long survival time of PPTID patients and the high frequency of the late neurotoxic side effects of irradiation, optimizing the irradiated area and the use of chemotherapy after surgery are important factors. ${ }^{30}$

Some chemotherapeutic agents can contribute to neurocognitive toxicity due to variable sensitivity of normal neural progenitor cells. ${ }^{11}$ A combination of epidermal growth factor receptor tyrosine kinase inhibitors and some proteasome inhibitors has been reported to have a potential risk of neurotoxicity compared to conventional chemotherapeutic agents. ${ }^{11}$

The development of radiation therapy and chemotherapy has contributed to the long-term survival of PPTID patients, even after recurrence. As such, combining systemic chemotherapy and craniospinal and whole-ventricular irradiation should be carefully considered to decrease severe late adverse effects.

\section{Leptomeningeal Dissemination}

Few reports are available regarding PC associated with leptomeningeal progression. Furthermore, details about the extent, time course, and treatment of diffuse widespread leptomeningeal dissemination of PC are largely unknown. ${ }^{9,15,26}$ Lutterbach et al. emphasized that survival time is strongly associated with CSF dissemination in adult PPT patients. Craniospinal control was found to be more effective in patients with PPTID. ${ }^{20,21}$

Stoiber et al. reported that local radiation therapy is successful in patients with PC and PPTID. ${ }^{28}$ Das et al. emphasized that radiation therapy was necessary for all cases of PPTID if complete resection was not achieved. Also, craniospinal irradiation would be a useful treatment for PC patients with leptomeningeal dissemination. ${ }^{7}$

Gomez et al. reported a patient in whom PC was diagnosed by biopsy and who was treated with local irradiation. Ten years after treatment, the patient showed craniospinal CSF dissemination, and histological examination of the recurrent tumor revealed a pathologically malignant change (MIB-1 index 10\%-15\%), and a diagnosis of PPTID was established. This patient received successful additional treatment with local and craniospinal irradiation and was without recurrence after 2 years of treatment. ${ }^{10}$

The efficacy of craniospinal and whole-ventricular irradiation in patients with PPTID has yet to be determined. However, Schild et al. investigated the important role of irradiation for patients with PPT in their analysis of 30 patients with pineal tumors and 105 with other germ cell tumors. ${ }^{25}$ They demonstrated a relationship between the radiation dose and long survival time in patients with PPT: the patients who received $>50$ Gy of radiation had a significantly higher 3-year survival rate than those who received lower doses of irradiation ( $94 \%$ vs $56 \%$, respectively; $\mathrm{p}=0.03$ ).

\section{Conclusions}

We reported a rare case of a PC patient with CSF dis- semination 6 years after resection; histopathological study of the recurrent tumor revealed a malignant change to PPTID. Our case demonstrates the importance of longterm follow-up of patients with PPT after resection and the efficacy of craniospinal irradiation in the treatment of leptomeningeal dissemination.

\section{References}

1. Anan M, Ishii K, Nakamura T, Yamashita M, Katayama S, Sainoo M, et al: Postoperative adjuvant treatment for pineal parenchymal tumour of intermediate differentiation. J Clin Neurosci 13:965-968, 2006

2. Bota DA, Alexandru D, Keir ST, Bigner D, Vredenburgh J, Friedman HS: Proteasome inhibition with bortezomib induces cell death in GBM stem-like cells and temozolomideresistant glioma cell lines, but stimulates GBM stem-like cells' VEGF production and angiogenesis. J Neurosurg 119:1415-1423, 2013

3. Brown WR, Blair RM, Moody DM, Thore CR, Ahmed S, Robbins ME, et al: Capillary loss precedes the cognitive impairment induced by fractionated whole-brain irradiation: a potential rat model of vascular dementia. J Neurol Sci 257:67-71, 2007

4. Cho BK, Wang KC, Nam DH, Kim DG, Jung HW, Kim HJ, et al: Pineal tumors: experience with 48 cases over 10 years. Childs Nerv Syst 14:53-58, 1998

5. Clark AJ, Ivan ME, Sughrue ME, Yang I, Aranda D, Han SJ, et al: Tumor control after surgery and radiotherapy for pineocytoma. J Neurosurg 113:319-324, 2010

6. Clark AJ, Sughrue ME, Ivan ME, Aranda D, Rutkowski MJ, Kane AJ, et al: Factors influencing overall survival rates for patients with pineocytoma. J Neurooncol 100:255-260, 2010

7. Das P, Mckinstry S, Devadass A, Herron B, Conkey DS: Are we over treating pineal parenchymal tumour with intermediate differentiation? Assessing the role of localised radiation therapy and literature review. Springerplus 5:26, 2016

8. Fangusaro J, Finlay J, Sposto R, Ji L, Saly M, Zacharoulis $\mathrm{S}$, et al: Intensive chemotherapy followed by consolidative myeloablative chemotherapy with autologous hematopoietic cell rescue (AuHCR) in young children with newly diagnosed supratentorial primitive neuroectodermal tumors (sPNETs): report of the Head Start I and II experience. Pediatr Blood Cancer 50:312-318, 2008

9. Fauchon F, Jouvet A, Paquis P, Saint-Pierre G, Mottolese C, Ben Hassel M, et al: Parenchymal pineal tumors: a clinicopathological study of 76 cases. Int J Radiat Oncol Biol Phys 46:959-968, 2000

10. Gomez C, Wu J, Pope W, Vinters H, Desalles A, Selch M: Pineocytoma with diffuse dissemination to the leptomeninges. Rare Tumors 3:e53, 2011

11. Gong X, Schwartz PH, Linskey ME, Bota DA: Neural stem/ progenitors and glioma stem-like cells have differential sensitivity to chemotherapy. Neurology 76:1126-1134, 2011

12. Hasegawa T, Kondziolka D, Hadjipanayis CG, Flickinger JC, Lunsford LD: The role of radiosurgery for the treatment of pineal parenchymal tumors. Neurosurgery 51:880-889, 2002

13. Hirato J, Nakazato Y: Pathology of pineal region tumors. J Neurooncol 54:239-249, 2001

14. Ito T, Kanno H, Sato K, Oikawa M, Ozaki Y, Nakamura H, et al: Clinicopathologic study of pineal parenchymal tumors of intermediate differentiation. World Neurosurg 81:783-789, 2014

15. Jouvet A, Saint-Pierre G, Fauchon F, Privat K, Bouffet E, Ruchoux MM, et al: Pineal parenchymal tumors: a correlation of histological features with prognosis in 66 cases. Brain Pathol 10:49-60, 2000 
16. Kurisaka M, Arisawa M, Mori T, Sakamoto T, Seike M, Mori $\mathrm{K}$, et al: Combination chemotherapy (cisplatin, vinblastin) and low-dose irradiation in the treatment of pineal parenchymal cell tumors. Childs Nerv Syst 14:564-569, 1998

17. Li G, Mitra S, Karamchandani J, Edwards MS, Wong AJ: Pineal parenchymal tumor of intermediate differentiation: clinicopathological report and analysis of epidermal growth factor receptor variant III expression. Neurosurgery 66:963-968, 2010

18. Limoli CL, Giedzinski E, Rola R, Otsuka S, Palmer TD, Fike JR: Radiation response of neural precursor cells: linking cellular sensitivity to cell cycle checkpoints, apoptosis and oxidative stress. Radiat Res 161:17-27, 2004

19. Louis DN, Ohgaki H, Wiestler OD, Cavenee WK, Burger PC Jouvet A, et al: The 2007 WHO classification of tumours of the central nervous system. Acta Neuropathol 114:97-109, 2007

20. Lutterbach J, Fauchon F, Schild SE, Chang SM, Pagenstecher A, Volk B, et al: Malignant pineal parenchymal tumors in adult patients: patterns of care and prognostic factors. Neurosurgery 51:44-56, 2002

21. Lutterbach J, Pagenstecher A, Spreer J, Hetzel A, Velthoven V, Nikkhah G, et al: The brain tumor board: lessons to be learned from an interdisciplinary conference. Onkologie 28:22-26, 2005

22. Redmond KJ, Mahone EM, Terezakis S, Ishaq O, Ford E, McNutt T, et al: Association between radiation dose to neuronal progenitor cell niches and temporal lobes and performance on neuropsychological testing in children: a prospective study. Neuro Oncol 15:360-369, 2013

23. Rickert CH, Simon R, Bergmann M, Dockhorn-Dworniczak B, Paulus W: Comparative genomic hybridization in pineal parenchymal tumors. Genes Chromosomes Cancer 30:99104,2001

24. Sato K, Kubota T: Pathology of pineal parenchymal tumors. Prog Neurol Surg 23:12-25, 2009

25. Schild SE, Scheithauer BW, Haddock MG, Wong WW, Lyons MK, Marks LB, et al: Histologically confirmed pineal tumors and other germ cell tumors of the brain. Cancer 78:25642571, 1996

26. Schild SE, Scheithauer BW, Schomberg PJ, Hook CC, Kelly PJ, Frick L, et al: Pineal parenchymal tumors. Clinical, pathologic, and therapeutic aspects. Cancer 72:870-880, 1993
27. Shimada K, Nakamura M, Kuga Y, Taomoto K, Ohnishi H, Konishi N: Cytologic feature by squash preparation of pineal parenchyma tumor of intermediate differentiation. Diagn Cytopathol 36:749-753, 2008

28. Stoiber EM, Schaible B, Herfarth K, Schulz-Ertner D, Huber PE, Debus J, et al: Long term outcome of adolescent and adult patients with pineal parenchymal tumors treated with fractionated radiotherapy between 1982 and 2003-a single institution's experience. Radiat Oncol 5:122, 2010

29. Tsumanuma I, Tanaka R, Washiyama K: Clinicopathological study of pineal parenchymal tumors: correlation between histopathological features, proliferative potential, and prognosis. Brain Tumor Pathol 16:61-68, 1999

30. Watanabe T, Mizowaki T, Arakawa Y, Iizuka Y, Ogura K, Sakanaka K, et al: Pineal parenchymal tumor of intermediate differentiation: treatment outcomes of five cases. Mol Clin Oncol 2:197-202, 2014

\section{Disclosures}

The authors report no conflict of interest concerning the materials or methods used in this study or the findings specified in this paper.

\section{Author Contributions}

Conception and design: Bando. Acquisition of data: Shinoda. Analysis and interpretation of data: Imai, Ichikawa. Critically revising the article: Ueno. Administrative/technical/material support: Mikami, Hori. Study supervision: Kuramoto, Kuroyama, Shimo, Matsumoto, Hirai.

\section{Supplemental Information}

\section{Previous Presentations}

Portions of this work were presented in abstract poster form at the 74th Annual Meeting of the Japan Neurosurgical Society, Sapporo, Japan, October 14, 2015.

\section{Correspondence}

Toshiaki Bando: Shinko Hospital, Kobe City, Japan. toshiakibando4@gmail.com. 